\title{
Cloud formation in substellar atmospheres
}

\author{
Christiane Helling \\ SUPA, School of Physics and Astronomy, Univ. of St Andrews, North Haugh, \\ St Andrews, KY16 9SS, UK
}

\begin{abstract}
Clouds seem like an every-day experience. But - do we know how clouds form on brown dwarfs and extra-solar planets? How do they look like? Can we see them? What are they composed of? Cloud formation is an old-fashioned but still outstanding problem for the Earth atmosphere, and it has turned into a challenge for the modelling of brown dwarf and exo-planetary atmospheres. Cloud formation imposes strong feedbacks on the atmospheric structure, not only due to the clouds own opacity, but also due to the depletion of the gas phase, possibly leaving behind a dynamic and still supersaturated atmosphere. I summarise the different approaches taken to model cloud formation in substellar atmospheres and workout their differences. Focusing on the phase-nonequilibrium approach to cloud formation, I demonstrate the inside we gain from detailed microphysical modelling on for instance the material composition and grain size distribution inside the cloud layer on a Brown Dwarf atmosphere. A comparison study on four different cloud approaches in Brown Dwarf atmosphere simulations demonstrates possible uncertainties in interpretation of observational data.
\end{abstract}

Keywords: astrochemistry, stars: atmospheres, stars: low-mass, brown dwarfs, infrared: stars PACS: 95.30.Wi, 97.10.Ex, 97.20.Vs

\section{INTRODUCTION}

Brown Dwarfs are object much more massive than planets, but they can become as cold as planets during their lifetime. Young gas giants and also so-called close-in giant gas planets can be as hot as Brown Dwarfs (e.g. [8], [16]). Brown Dwarfs can only nucleosynthesis energy during a short period of their lift time, which is enough to bust their luminosity to a level where they can be much easier observed than any planet at a similar distance. The largest similarity between Brown Dwarfs and planets is the rich, multi-phase atmospheric chemistry. Descending the main sequence makes Brown Dwarfs and possibly late-type M-dwarfs the only objects, that form like stars and that contain dust (i.e. small solid particles) in their atmosphere. After this discovery [23], it became immediately clear that Brown Dwarfs were not the perfect example for a static atmosphere but feedback of the dust clouds on the atmospheric structure, the chemistry, and the radiative transfer would need attention. Other places of efficient dust formation are the circulstellar envelopes of AGB stars which are extremely dynamic systems due to the presence of dust (see review by A.C. Andersen, and contributions by e.g. S.Höfner, N. Thureau this volume). [10] have presented spectra for L-type Brown Dwarfs showing broad absorption features between $8 \mu \mathrm{m}$ and $11 \mu \mathrm{m}$ which is the wavelength range were silicate dust would absorb. Although the idea of dust absorbing in the atmosphere is thermodynamically correct, it is hard to proof its existence directly since we are basically searching for the absorption signature of a thin transparent layer on top of an optically thick wall ([19]). [20] concluded that inhomogeneities in cloud decks and the evolution 


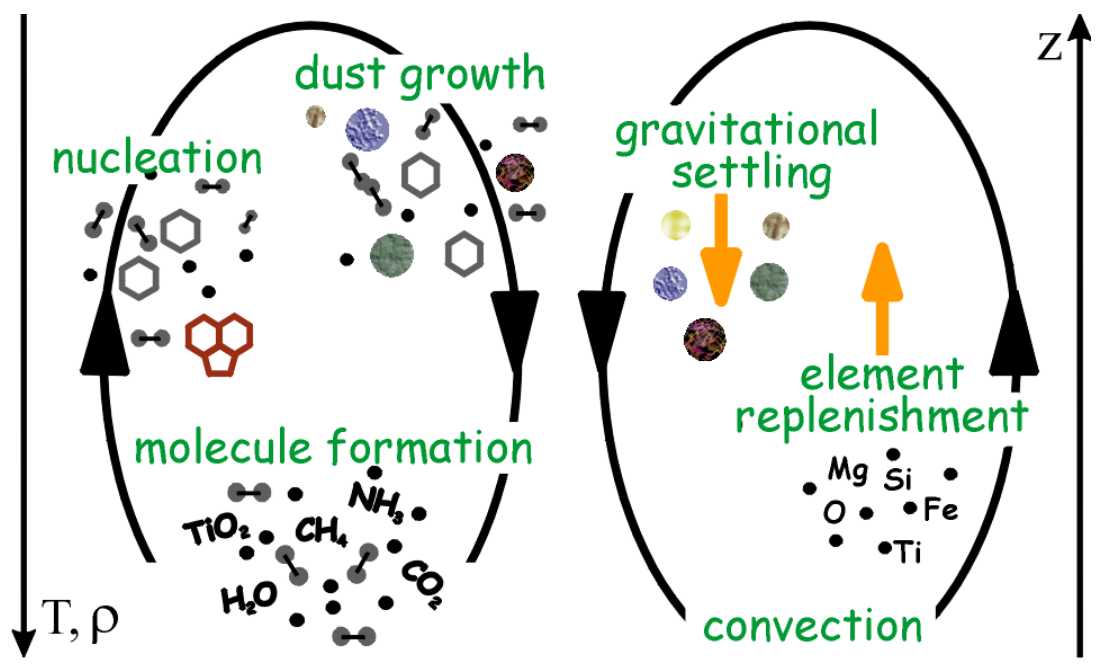

FIGURE 1. Interaction of multi-scale processes during cloud formation in Brown Dwarf atmospheres.Dust formation and turbulence act on small scales, and gravitational settling and element replenishment act on large scales.

thereof can plausibly produce observed photometric $I$-band variations. Extensive studies searching for uncorrelated, time-dependent variability due to a variable cloud coverage of the atmosphere, suggest a variability of 2-3\% ([14]) or even 2-10\% ([11]) depending in the wavelength studied. Richardson et al. (2007) infer the presence of silicate haze based on secondary-eclipse Splitzer observations on giant gas-planets, a conclusion which was also put forward by [29] based on HST transmission spectra for HD 189733b (see also reviews by G. Tinetti and by J. Harrington, this volume).

\section{CLOUD FORMATION PROCESSES}

Three major complexes need to be modelled to account for cloud formation in model atmospheres and to produce observable informations. Figure 1 demonstrates how condensation, gravitational settling and element replenishment interact.

\section{- Condensation:}

Seed particle form from the gas phase via a net or a path of chemical reaction. Once the first solid surface appears from the gas phase, chemical surface reaction can proceed rapidly to grow a massive mantle such that the initially tiny seed growth to a macroscopic grain of $\mu \mathrm{m}$-size. While the nucleation process is only possible in a highly supersaturated gas, the growth can take place if a solid is thermally stable. The counter-acting process is evaporation if the temperature becomes too high. The condensation process reduces the element abundances is the gas phase selectively regarding those element forming grain monomers (like $\mathrm{MgSiO}_{3}, \mathrm{TiO}_{2}, \mathrm{SiO}_{2}$ in the $\mathrm{MgSiO}_{3}[\mathrm{~s}]-, \mathrm{TiO}_{2}[\mathrm{~s}]-, \mathrm{SiO}_{2}[\mathrm{~s}]$-lattice etc.).

- Gravitational settling

Once the grains are present they start to fall into the atmosphere due to the strong 
gravitational attraction in Brown Dwarfs and in planets. Since the grain sizes are not constant but different grain sizes will appear with different grain numbers, the equation of motion would need to be solved for a grain size distribution. An easier case is to solve the equation of motion for a height-dependent mean grain size derived from a grain size distribution.

- Element replenishment

A static atmosphere would be dust free ([41]). [27], [36] and [25] infer from their observations that convection mixes up (elementally rich) gas from inner and hotter atmospheric regions, a mechanism essential for allowing the dust cloud to persist inside the atmospheres. The friction of the gas fluid is not very large so that its inertia drives it forward once it has been set into motion. Such fluids are called turbulent where whirly motions exist on a large range of scale. Convection is just one of them, but the largest on which the turbulent fluid field is energetically generated. Hence, modelling element replenishment means to describe mixing, advection, overshooting, and turbulence.

As soon as the first Brown Dwarf was discovered, model atmosphere predictions where needed, and three different ideas appeared regarding the phenomenology of cloud formation:

i) Static: The cloud particle are so small that they remain where they formed. (Tsuji, Copper et al.),

ii) Bottom-up: The cloud forms by up-mixing of uncondensed material. (Ackerman \& Marley, Allard et al.)

iii) Top-down: The cloud is formed by particles falling into the atmosphere and evolving on their way downward. (Helling \& Woitke)

Table 1 summarises the cloud models and their variants presently applied in the literature. The grain size is an opacity input quantity, and the very first models set it constant assuming the grains to hoover inside the atmosphere (Tsuji). Later, experiences from cloud observations on Earth led to a time-scale comparisons to derive the grain sizes (Allard et al.) or to an analytic parameterisation thereof (Cooper et al., Ackerman \& Marley). Only very recently, a grain size distribution based on a model for dust formation was derived (Helling \& Woitke). The calculation of the dust cloud opacity needs further the material composition of the cloud. The modelling of this is very much linked to the model assumption regarding the formation process. Almost all models assume phase-equilibrium between the atmospheric gas and the cloud particles, hence the supersaturation ratio $S=1$, or just a $1 \%$ supersaturation. However, only the departure from this equilibrium state, i.e. $S \gg 1$, would allow the actual grain formation. Assuming phase-equilibrium allows to consider the thermal stability of a vast variety of potential condensates (e.g. [26]) which then can be considered to deplete the gas-phase. This phase-equilibrium approach bears the danger that one considers compounds which might never have a chance to actually form.

Despite the variety of the assumptions made to model clouds in Brown Dwarf atmospheres (Table 1), fundamental understanding has been added by considering the limiting cases case B / dusty and case C / cond by Tsuji and Allard et al., respectively. The dust is element sink and opacity source inside the atmospheres in case B / dusty which 
TABLE 1. Cloud models in Brown Dwarf atmosphere simulations.

\begin{tabular}{|c|c|c|c|c|c|}
\hline \multirow[b]{2}{*}{ Author } & \multicolumn{3}{|c|}{ Assumptions } & \multirow{2}{*}{\multicolumn{2}{|c|}{ Model variants }} \\
\hline & grain size $a$ & $\begin{array}{l}\text { grain } \\
\text { composition }\end{array}$ & $\begin{array}{l}\text { super- } \\
\text { saturation }\end{array}$ & & \\
\hline $\begin{array}{l}\text { Tsuji } \\
\text { (also Burrows 2001) }\end{array}$ & $a=10^{-2} \mu \mathrm{m}$ & homog. & $S=1$ & $\begin{array}{l}\text { case B } \\
\text { case } C \\
U C M\end{array}$ & $\begin{array}{l}\text { full dusty model } \\
\text { dust cleared model } \\
\text { dust between } \\
T_{\text {cr }}<T<T_{\text {cond }} \\
\text { [39], [40], [38], [37] }\end{array}$ \\
\hline Allard \& Homeier & $\begin{array}{l}f(a)=a^{-3.5} \\
\text { time scales dep. }\end{array}$ & $\begin{array}{l}\text { homog. } \\
\text { homog. }\end{array}$ & $\begin{array}{l}S=1 \\
S=1.001\end{array}$ & $\begin{array}{l}\text { dusty } \\
\text { cond } \\
\text { settl }\end{array}$ & $\begin{array}{l}\text { full dusty model } \\
\text { dust cleared model } \\
\text { time scales } \\
{[2],[3],[4],[33]}\end{array}$ \\
\hline Copper et al. & $\begin{array}{l}f(a) \sim\left(\frac{a}{a_{0}}\right)^{6} \\
\times \exp \left[-6\left(\frac{a}{a_{0}}\right)\right]\end{array}$ & homog. & $S=1.001$ & & [9] \\
\hline $\begin{array}{l}\text { Ackerman \& Marley } \\
\& \text { Lodders }\end{array}$ & log-normal $f(a, z)$ & homog. & $S=1$ & $f_{\text {sed }}$ & $\begin{array}{l}\text { sedimentation } \\
\text { efficiency [1] }\end{array}$ \\
\hline Helling \& Woitke & $f(a, z)$ & dirty & $S=S(z, s)$ & & [42], [18] \\
\hline
\end{tabular}

represents the the mid-L-dwarf regime. Case C / cond do consider the dust as element sink only and neglect its opacity. This case represented the T-dwarfs regime very well. Hence, these two simple limiting cases have established our picture from L-dwarfs as being covered in thick clouds, and of the T-dwarfs as Brown Dwarfs with an elementally depleted atmosphere but without visible clouds. As question remains how the clouds disappear going from L- to T-dwarfs (L-T transition region, [24], [15]). The main idea is that the cloud thickness changes. This is parameterised by a critical temperature $T_{\mathrm{cr}}$ ([38]), or a sedimentation efficiency $f_{\text {sed }}$ ([1],[10]), or the mixing efficiency (Allard et al.). As long as no consistent theory exists to allow the simulation of the L-T, such empirical parameterisations are needed and modellers try to constrain them from observations ([37]; Stephens et al., this volume). This inconsistency complicates also the evolutionary modelling from the L- into the T-dwarf regime as discussed in [34].

\section{THE PHASE-NON-EQUILIBRIUM APPROACH}

The cloud formation process can only proceed in non-equilibrium state, else the system would not change at all. A phase-transition will therefore require a phase-nonequilibrium, hence, the gas phase needs to be supersaturated $(S \gg 1)$. Depending on time and grain size scales, the dust formation proceeds via 1.) nucleation, followed by 2.) growth (Sect. $)$. Nucleation runs on much shorter time scales than the growth process and needs a highly supersaturated gas to allow a reaction efficiency high enough to counterbalance destructive backward reactions. The (not-yet) particles which subsequently form in the nucleation regime are macro-molecules and clusters (e.g. [13, 28]). The result is a nucleation rate which determines the number of dust particles. [22, 21] have shown that $\mathrm{TiO}_{2}$ is a suitable candidate for seed formation in oxygen-rich environments since enough molecular $\mathrm{TiO}_{2}$ is available to allow an efficient homogeneous cluster forma- 

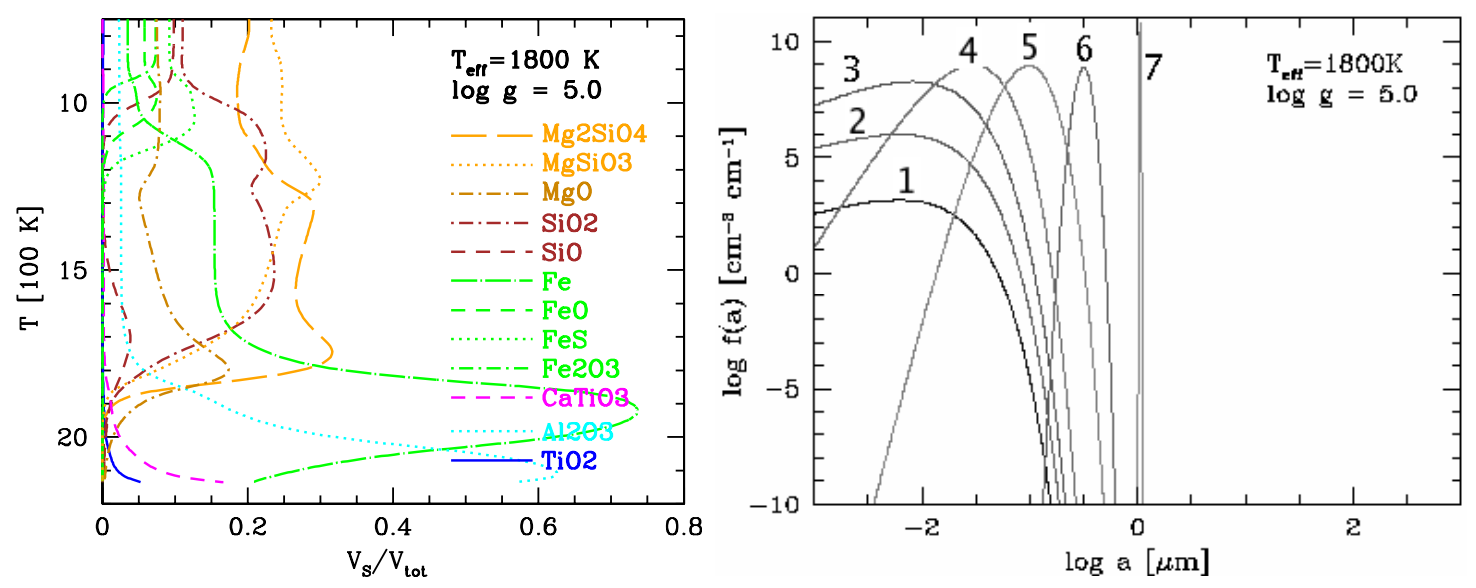

FIGURE 2. Material composition (in volume fractions $V_{\mathrm{s}} / V_{\text {tot }}$; left) and altitude-dependent grain size distributions $\left(f(a)\right.$ in $\mathrm{cm}^{-3} \mathrm{~cm}^{-1}$; right) in a stationary dust cloud layer in a Brown Dwarf atmospheres.

tion process, in fact much more efficient than for instance SiO- or Fe-nucleation. Once, such a first surface emerges from the gas phase, lots of other compounds are already thermally stable and can therefore grow by chemical surface reactions. Still, thermal stability is not the only criterion, but we need to know which chemical surface reactions are possible (see [18]). A straight forward observation from gas-phase chemical equilibrium calculations is, that only small molecules like $\mathrm{MgO}, \mathrm{MgOH}, \mathrm{SiO}, \mathrm{SiS}, \mathrm{Al}_{2} \mathrm{O}$, $\mathrm{Ca}(\mathrm{OH})_{2}$ are abundant enough to allow high growth rates. This conclusion is supported by condensation experiments [31, 32].

The treatment of $\mathrm{TiO}_{2}$-nucleation by a modified classical nucleation theory [21], and the calculation of the grow process including the effect of drift in connection with a parametrised up-mixing of uncondensed gas $([42,41,19,18])$ allows to study the cloud's material composition, the altitude-dependent grain size distribution, and also the phaseequilibrium state of the dust-gas mixture in the cloud beside various other details of the dust complex like nucleation rate, growth velocity, dust-to-gas ratio, metallicity. Figure 2 (left) shows that the cloud deck is populated by silicate grains mainly made of $\mathrm{MgSiO}_{3}[\mathrm{~s}]$ and $\mathrm{Mg}_{2} \mathrm{SiO}_{4}$ [s] beside some iron-compounds. The $\mathrm{MgSiO}_{3}$ [s] drops with increasing temperate which increases the fraction of $\mathrm{SiO}_{2}[\mathrm{~s}]$ inside $\approx 1000 \mathrm{~K}$. All silicates are evaporated at $T \approx 2000 \mathrm{~K}$, and the cloud particles are now composed of mainly $\mathrm{Fe}[\mathrm{s}]$, and $\mathrm{Al}_{2} \mathrm{O}_{3}[\mathrm{~s}]$ at even higher temperatures. Figure 2 (right) shows a number of selected grain size distribution function, $f(a)\left[\mathrm{cm}^{-3} \mathrm{~cm}^{-1}\right]$, sampling the cloud layer of that model. The lowest curve (1) represent the outermost grain size distribution in the region of nucleation of those depicted here. All subsequent $f(a)(2-7)$ move towards larger grain sizes (abscissa) but only numbers 1, 2, 3 show an increase in numbers of grains. 4, 5, 6 have achieved the maximum possible number of grains and only move in grain-space to the right, hence, they represent cloud layers with continuously increasing grain sizes. 4, 5, 6 also narrower which demonstrates that the cloud base is populates by a relatively narrow interval of grain sizes. These big grains are mainly made of $\mathrm{Fe}[\mathrm{s}]$, or $\mathrm{Al}_{2} \mathrm{O}_{3}[\mathrm{~s}]$ just before they evaporate.

The phase-non-equilibrium approach discussed so far does not assume a certain 


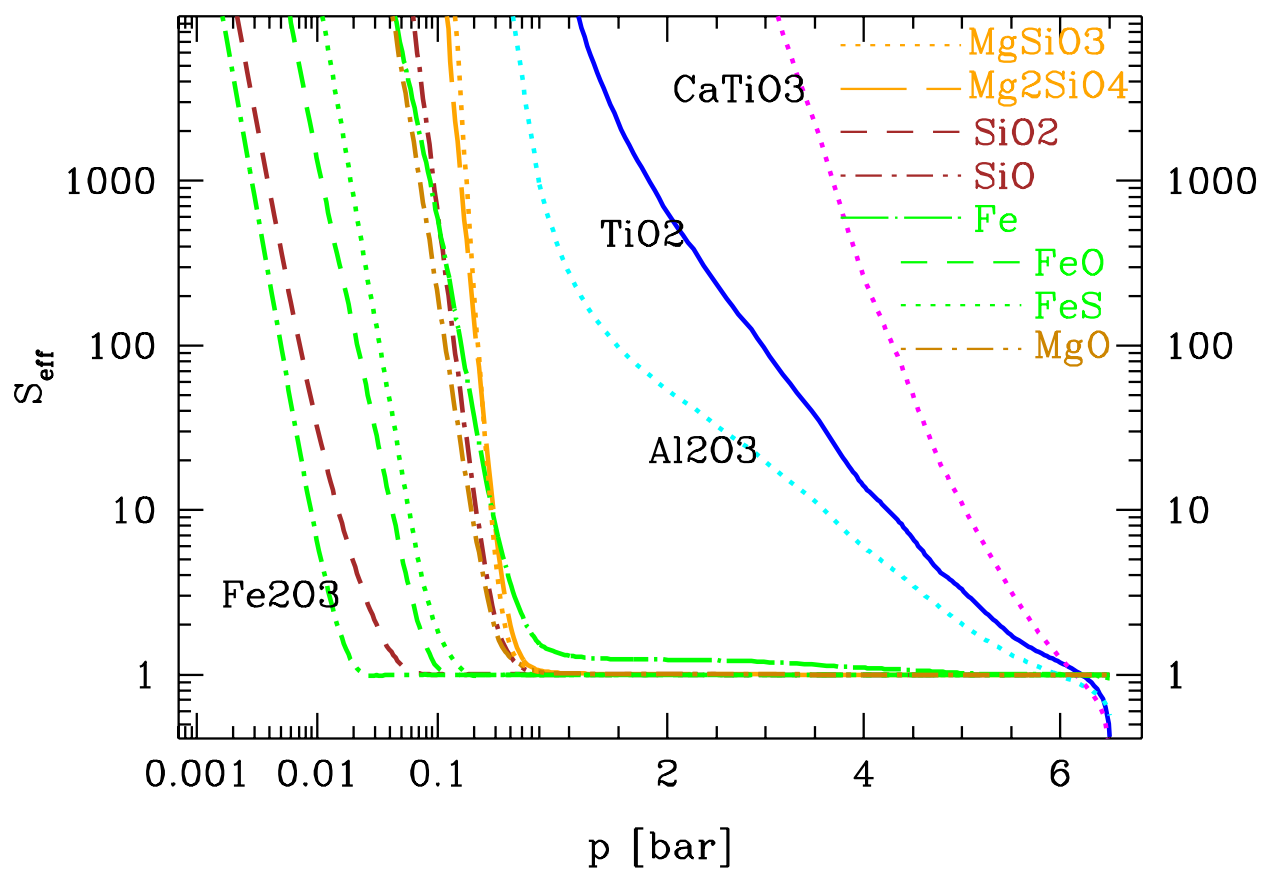

FIGURE 3. Effective supersaturation ratio $\left(S_{\text {eff }}\right)$ of the dust compounds considered in Fig. 22 Note that $S_{\text {eff }}$ has been defined in (Helling, Woitke \& Thi 2008) to discuss the contribution of all growth reaction of a particular compound compared to the standard definition.

supersaturation ratio, $\mathrm{S}$, but calculates it according to the present thermodynamical conditions and element abundances. We are therefore in the position to study if and where the dust-gas mixture forming the cloud layer is in phase equilibrium or not. Hence, we can study where the cloud is thermally stable $(S=1)$ and where is is effectively processed in a constructive $(S>1)$ or destructive $(S<1)$ way. Figure 3 depicts the effective supersaturation ratio, $S_{\text {eff }}$, defined to take into account the contribution of all growth reaction for a particular compound. The compounds considered fall into two regimes:

- rare-element compounds:

Ti-, Al-, Ca-solids exhibit an extreme phase-non-equilibrium

- abundant-element compounds:

$\mathrm{Mg}$-, Si-, Fe-solids achieve phase-equilibrium over some pressure scale height in the cloud once the seed particles serve as condensation surface

Figure 3 shows that also Fe[s] remains in phase-non-equilibrium in almost the entire cloud layer, hence only the low-temperature condensates can achieve phase-equilibrium.

\footnotetext{
1 The classical supersaturation ratio assumes that the compound monomer (like a $\mathrm{Mg}_{2} \mathrm{SiO}_{4}$-molecule for $\mathrm{Mg}_{2} \mathrm{SiO}_{4}[\mathrm{~s}]$-solid) does exist in the gas phase. Since this is not always the case, the supersaturation ratio for every surface reaction needs to be calculated since here the gas-phase constituents are known. For further details, see [19].
} 
TABLE 2. Brown Dwarf model atmosphere codes in comparison. See also Table 1 s - solid, sl - solid \& liquid.

\begin{tabular}{|c|c|c|c|c|c|}
\hline authors & $\begin{array}{l}\text { element } \\
\text { abund. }\end{array}$ & elements & $\begin{array}{l}\text { gas-phase } \\
\text { spec. }\end{array}$ & $\begin{array}{l}\text { number of } \\
\text { dust species }\end{array}$ & \\
\hline Tsuji & {$[\underline{6}]$} & 34 & 83 & $\begin{array}{l}3 \text { as opacity source } \\
10 \text { as element sinks }\end{array}$ & $\begin{array}{l}\mathrm{s} \\
\mathrm{s}\end{array}$ \\
\hline Allard \& Homeier & {$[12]$} & 84 & 680 & $\begin{array}{l}43 \text { as opacity source } \\
169 \text { as element sinks }\end{array}$ & $\begin{array}{l}\text { sl } \\
\text { sl }\end{array}$ \\
\hline $\begin{array}{l}\text { Marley, Ackerman } \\
\text { \& Lodders }\end{array}$ & [26] & 83 & $\sim 2200$ & $\begin{aligned} & 5 \text { as opacity source } \\
\sim & 1700 \text { as element sinks }\end{aligned}$ & $\begin{array}{c}\mathrm{s} \\
\mathrm{sl}\end{array}$ \\
\hline $\begin{array}{l}\text { Dehn \& Hauschildt } \\
\text { + Helling \& Woitke }\end{array}$ & [12] & 40 & 338 & $\begin{array}{l}7 \text { as opacity source } \\
7 \text { as element sinks }\end{array}$ & $\mathrm{s}$ \\
\hline
\end{tabular}

Consequently, phase-non-equilibrium models would show larger gas-phase abundances regarding molecules containing element of thermally very stable compounds as demonstrated in [17].

\section{COMPARING DUST CLOUD MODELS}

"Where stability and equilibrium are inherent signs if an unchanging and 'static' world, the formation and evolution of complex physical systems is intimately coupled to the existence and stabilisation of non-equilibrium states of matter." [28].

Section ${ }_{\square}$ already hints that present cloud models are well described by the above statement taken from [28]. Namely, one kind of approaches studies the possible endstate of the cloud formation processes which is the (phase-)equilibrium state, the other kind has its emphasis on the formation process which is the (phase-)non-equilibrium state. Both aim to understand the influence of atmospheric dust clouds on observable quantities by means of model atmospheres simulations. A comparison study of such models with emphasis on the dust approaches was initiated at a workshop in Leiden 2006 22). Table 2 demonstrates that not only the cloud models are different (Table 1) but also the way the results of the cloud model approaches are used in the radiative equilibrium calculation differ. The number of compounds considered as opacity species is usually smaller than the number of compounds used as element sinks, and the element abundance data are taken from different sources. Part of the reason are missing refractory index data and numerical difficulties in handling such complex absorbing systems. The element abundances are particular interesting since they determine the composition of the gas phase (beside the local temperature and density) from which the dust forms.

Figure 4 compares the results from four different model atmospheres codes (Table 2) for the stellar parameter combination of a typical solar-metallicity L-dwarf. The

2 http://www.lorentzcenter.nl/lc/web/2006/203/info.php3?wsid=203 


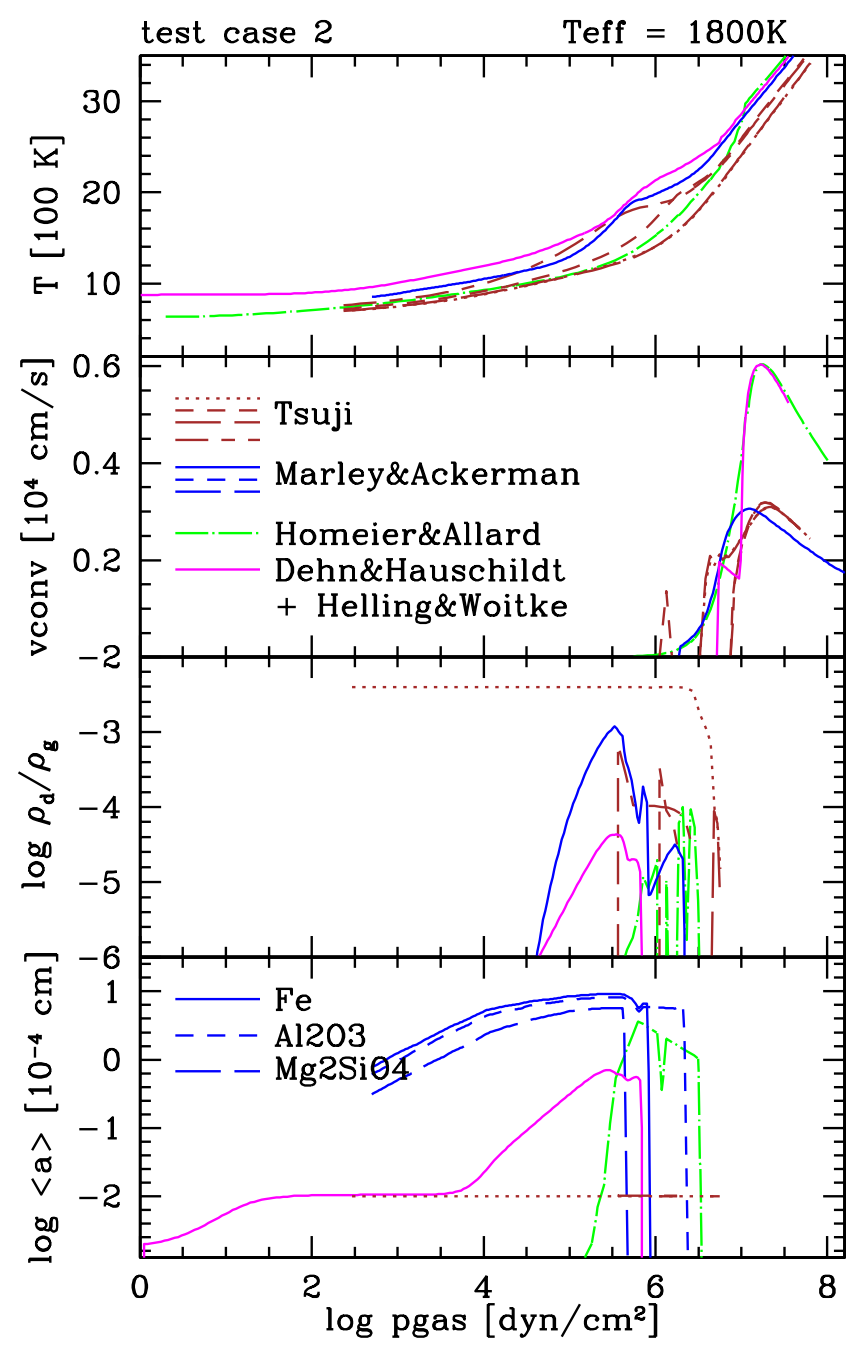

FIGURE 4. Test cases for complete atmospheric models for $\log g=5.0$, solar element abundance with $\mathrm{T}_{\text {eff }}=1800 \mathrm{~K}$. Note: Different colours stand for different stellar atmospheres codes. Four models are plotted for the Tsuji-case (brown): long-short-dashed: $T_{\mathrm{cr}}=1700 \mathrm{~K}$ (extended cloud), short-dashed: $\mathrm{T}_{\mathrm{cr}}=1800 \mathrm{~K}$, long-dashed: $\mathrm{T}_{\mathrm{cr}}=1900 \mathrm{~K}$ (thin cloud), dotted: no dust opacity considered. Different line styles in $\log \langle a\rangle$ indicate different homogeneous dust species in the Marley, Ackerman \& Lodders-models.

temperature-pressure structures, $(T, p)\left(1^{\text {st }}\right.$ panel $)$, differ the most in the low-pressure regime above the cloud layer and in the $(T, p)$-range where the cloud sits. The models which produce a thick cloud layer show the backwarming effect due to the strong cloud opacity at $\approx 2000 \mathrm{~K}$. The dust-to-gas mass ratio, $\rho_{\mathrm{d}} / \rho_{\mathrm{g}}\left(3^{\text {rd }}\right.$ panel), demonstrates where most of the dust mass is located, and coincides with the backwarming effect. However, the $\rho_{\mathrm{d}} / \rho_{\mathrm{g}}$ maxima differ widely amongst the models. Such a strong difference must be attributed to the amplification of small differences in a coupled system such as a stellar atmosphere, because Helling et al. (2008) demonstrate much smaller differences in the $\rho_{\mathrm{d}} / \rho_{\mathrm{g}}$ maxima if the dust models alone are tested. The models also differ in the predicted mean grain sizes, $\langle a\rangle$ ( $4^{\text {th }}$ panel), which reflects the differences in the cloud model approaches the strongest amongst the quantities in Fig. 4. 


\section{CONCLUSIONS}

The modelling of Brown Dwarf atmospheres has turned into an unexpected challenge as these atmosphere contain dust which causes strong feedbacks as opacity source and element sink. Different approaches are applied to model dust in Brown Dwarf atmospheres, and obeying its nature as coupled system of nonlinear equation, atmosphere simulations applying different cloud models do produce different results although the over-all cloud-structure appears robust. A fundamental difference amongst the models is the consideration of phase-equilibrium. Kinetic models did show that in particular compounds made or rare elements like $\mathrm{Ti}, \mathrm{Al}$, and $\mathrm{Ca}$ never achieve phase equilibrium, i.e. thermal stability, in the Brown Dwarf atmosphere. Furthermore, the transition from the L- into the T-dwarf regime is not understood in enough detail that consistent atmosphere simulations are available. This is a sever challenge for evolutionary models.

What else needs to be done? A still outstanding problem is the modelling of turbulent dust formation in large scale simulations which is also of significance for planetary atmospheres or protoplanetary disks. The challenge is here not to lose the small-scale interactions between the chemistry and the fluid field which has been shown to support the turbulent nature of the fluid field and to even cause the appearance of medium-scale cloud structures. A second challenge is the possible coupling of the dust-ionised atmosphere to the object's magnetic field. [35] and [20] argue against an ionisation potential in Brown Dwarf atmospheres. However, drifting grains in a turbulent environment might make a re-consideration necessary.

\section{REFERENCES}

1. A. Ackerman, M. Marley 2001, ApJ 556, 872

2. F. Allard, P.H. Hauschildt, D.R. Alexander, A. Tamanai, A. Schweitzer, 2001, ApJ 556, 357

3. F. Allard, T. Guillot, H.-G. Ludwig, P.H. Hauschildt, A. Schweitzer, D.R. Alexander, J.W. Ferguson 2003, IAU 211, 324

4. F. Allard, N.F. Allard, D. Homeier, J. Kielkopf, M.J. McCaughrean, F. Spiegelman 2007, A\&A 474, L21

5. C. Allende Prieto, D.L. Lambert, M. Asplund 2002, ApJ 573, 137

6. E. Anders, N. Grevesse 1986, Ceochim.Cosmochim.Acta 53, 197

7. M. Asplund, N. Grevesse, A.J. Sauval 2005, APS Conf. Series 336, 25

8. A. Collier-Cameron, D. Pollacco, C. Hellier et al. 2008, F. Pont, ed., IAU 253, in press

9. C.S. Cooper, D. Sudarsky, J.A. Milsom, J.I. Lunine, A. Burrows 2003, ApJ 586, 1320

10. M.C. Cushing, M.S. Marley, D. Saumon et al. 2008, ApJ 678(2), 1372

11. C.A.L. Bailer-Jones 2008, MNRAS, 384, 1145

12. N. Grevesse, A. Noels, A.J. Sauval 1992, ESA Proc. Coronal Streamers, Coronal Loops, and Coronal and Solar Wind Composition, 305

13. A. Goeres 1993, Rev, Mod. Astr. 6, 165

14. B. Goldman, M.C. Cushing, M.S. Marley et al. 2008, $A \& A, \mathbf{4 8 7}, 277$

15. D.A. Golimowski, S.K. Leggett, M.S. Marley, X. Fan, T.R. Geballe et al. 2004, ApJ, 127, 3516

16. L. Hebb, A. Collier-Cameron, D. Pollacco, R. Street, R. West 2008, MNRAS, in prep.

17. Ch. Helling, A. Ackerman, F. Allard, M. Dehn, P. Hauschildt, D. Homeier, K. Lodders, M. Marley, R. Rietmeijer, T. Tsuji, P. Woitke 2008, MNRAS, submitted

18. Ch. Helling, P. Woitke, W.-F. Thi 2008, $A \& A, \mathbf{4 8 5}, 547$

19. Ch. Helling, W.-F. Thi, P. Woitke, M. Fridlund 2006, $A \& A$, 451, L9

20. C.R. Gelino, M.S. Marley, J.A. Holtzman, A.S. Ackerman, K. Lodders 2002, ApJ, 577, 433

21. K.S. Jeong, C. Chang, E. Sedlmayr,D. Sülzle, 2000, J. Phys. B, 33, 3417 
22. K.S. Jeong, J.M. Winters, A.J. Fleischer, E. Sedlmayr, 1996, ASPC, 135, 335

23. H.R.A. Jones, and T. Tsuji 1997 ApJ, 134, 423

24. G.R. Knapp, S.K. Leggett, X. Fan, M.S. Marley, T.R. Geballe et al. AJ, 127, 3553

25. S.K. Leggett, D. Saumon, M.S. Marley, T.R. Geballe, D.A. Golimowski et al. 2007, ApJ, 655, 1079

26. K. Lodders 2003, ApJ, 591, 1220

27. B.R. Oppenheimer, S.R. Kulkarni, K. Matthews, M.H. van Kerkwijk 1998, ApJ 502, 932

28. A.B.C. Patzer 1998, PhD Thesis, TU Berlin, Germany

29. F. Pont, H. Knutson, R.L. Gilliland, C. Moutou, D. Charbonnneau 2008, MNRAS, 385, 109

30. L.J. Richardson, D. Deming, K. Horning, S. Seager, J. Harrington 2007, Nature 445, 892

31. F.J.M. Rietmeijer, J.A. Nuth, J.M. Karner 1999, ApJ, 527, 395

32. F.J.M. Rietmeijer, A. Pun, Y. Kimura, J.A. Nuth III 2007, Icar, 195, 493

33. W.B. Rossow 1978, Icarus 36, 1

34. D. Saumon, M.S. Marley 2008, ApJ (arXiv:0808.2611)

35. S. Mohanty, G. Basri, F. Shu, F. Allard, C. Chabrier 2002, ApJ, 571, 469

36. D. Saumon, M.S. Marley, S.K. Leggett, T.R. Geballe, D. Stephens et al 2007, ApJ 656, 1136

37. T. Tsuji 2005, ApJ 621, 1033

38. T. Tsuji 2002, ApJ 575, 264

39. T. Tsuji 2000, eds. R. Rebolo and M. R. Zapatero-Osorio, 156

40. T. Tsuji, Ohnaka K., Aoki W., Nakajima T. 1996b, A\&A 308, L29

41. P. Woitke, Ch. Helling 2007, A\&A 414, 335

42. P. Woitke, Ch. Helling 2003, A\&A 399, 297 